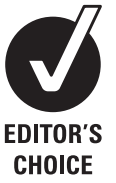

ennent Institute of Ophthalmology, Gartnavel General Hospital, Glasgow, UK

Correspondence to Heather C Russell, Tennent Institute of Ophthalmology, Gartnavel General Hospital, 1053 Great Western Road, Glasgow G12 OYN, UK; heatherrussell74@googlemail. com

Accepted 7 January 2010 Published Online First 8 June 2010

\title{
Topical mitomycin C chemotherapy in the management of ocular surface neoplasia: a 10-year review of treatment outcomes and complications
}

\author{
H C Russell, V Chadha, D Lockington, E G Kemp
}

\begin{abstract}
Introduction The use of topical mitomycin C (MMC) has gained popularity in the management of ocular surface neoplasia. The aim of this study is to determine outcomes and complications following such treatment. Methods This study is a retrospective review of patients treated with topical MMC for ocular surface neoplasia, including primary acquired melanosis (PAM), melanoma, corneal-conjunctival intraepithelial neoplasia (CCIN), squamous cell carcinoma (SCC) and sebaceous gland carcinoma (SGC). Data regarding diagnosis, short- and long-term outcomes, and short- and long-term complications, were recorded.
\end{abstract}

Results 58 patients were identified, with a mean age of 63 years and mean follow-up of 36 months. 21 received MMC as primary therapy and 37 as surgical adjuvant. The regimen was $0.04 \%$ MMC four times a day for 3 weeks on, 3 weeks off, 3 weeks on, with topical steroid and lubricants throughout. Initial clinical response was either partial or complete in 93\%. Overall, $26 \%$ developed recurrent disease at a mean of 13 months post treatment. Recurrence rates by pathology were 20\% PAM, 25\% melanoma, 0\% CCIN, $67 \%$ SCC and 57\% SGC. Short-term complications occurred in $52 \%$, but only $7 \%$ required treatment cessation. Long-term complications such as persisting keratoconjunctivitis, epiphora and corneal problems, occurred in $31 \%$.

Conclusion The results confirm the effectiveness of topical MMC chemotherapy in the management of ocular surface neoplasia. Self-limiting short-term complications were common; however, limbal stem cell deficiency appears to be a significant long-term complication of treatment, occurring in $12 \%$.

Mitomycin C (MMC) is an antibiotic isolated from Streptomyces caespitosus. It undergoes metabolic activation to become an alkylating agent that is cytotoxic to both proliferating and non-proliferating cells. ${ }^{1}$ Over the past 16 years, topical use of MMC has gained popularity in the management of ocular surface neoplasia, with the "whole eye treatment" offering potential benefits over excision and cryotherapy for the treatment of extensive and subclinical disease.

Primary acquired melanosis is a superficial patchy pigmentation of the conjunctiva that can lead to conjunctival melanoma in up to $30 \%$ of affected patients. $^{2}$ Topical MMC chemotherapy as a primary treatment has been found to be very effective in reducing and even eliminating pigmentation, with recurrence rates ranging from $13 \%$ to $38 \% .^{2-4}$ The role of topical MMC in the management of conjunctival melanoma is however, less clear, with reported recurrence rates of $33-100 \%$ for primary treatment ${ }^{3-5}$ and $0-66 \%$ for adjuvant treatment. ${ }^{4-6}$

Ocular surface squamous neoplasia is a rare condition with a range of clinical presentations from corneal-conjunctival intraepithelial neoplasia (CCIN) with no frank invasion, to invasive squamous cell carcinoma (SCC). ${ }^{7}$ Following the first report of use of topical MMC in the treatment of CCIN, ${ }^{8}$ many other studies have confirmed its effectiveness as both a primary and adjuvant therapy, with very low recurrence rates of $0-22 \% .^{9-12}$ It is also effective in the management of minimally elevated SCC, with recurrence rates as low as zero, ${ }^{13} 14$ and as a neoadjuvant "chemoreductant" followed by surgical excision for thicker tumours. ${ }^{15}$

Sebaceous gland carcinoma (SGC) is a rare tumour most commonly affecting the upper lid. Due to its propensity to pagetoid spread within the conjunctiva, the management of this tumour is notoriously difficult, often necessitating exenteration. ${ }^{16}$ Favourable results have been reported with use of topical MMC as an adjuvant in the management of SGC with pagetoid spread. ${ }^{17-19}$

Reported complications of topical MMC chemotherapy range from common transient self-limiting problems such as local irritation, hyperaemia, epiphora, allergy, keratoconjunctivitis and corneal abrasion, ${ }^{8} 141720$ to longer-lasting problems such as punctal stenosis, cataract, persisting keratoconjunctivitis and limbal stem cell deficiency. ${ }^{20-23}$

The purpose of this report is to describe our experience with topical MMC in the management of ocular surface neoplasia over the last 10 years. Data regarding treatment response, disease recurrence, and short- and long-term complications are presented.

\section{METHODS}

A retrospective case note review was performed of all patients with a diagnosis of primary acquired melanosis (PAM), conjunctival melanoma, CCIN, conjunctival/corneal SCC and SGC, who had attended the ocular oncology service at a single centre between January 1998 and February 2009.

The majority of patients received the treatment regimen of one drop of $0.04 \%$ topical MMC four times a day for 3 weeks, with a 3 -week period off, followed by another 3 weeks of MMC four times a day, regardless of whether treatment was primary, neoadjuvant or postsurgical adjuvant. For those receiving postsurgical adjuvant treatment, the 
Table 1 Summary of the indications for treatment by diagnosis

\begin{tabular}{lcclccc}
\hline Indications & PAM & Melanoma & CCIN & SCC & SGC & Total \\
\hline Primary treatment & 15 & 1 & 2 & & 3 & 21 \\
Neoadjuvant & 1 & 3 & 3 & 2 & 2 & 11 \\
Intraoperative adjuvant & & 3 & & & 1 & 4 \\
Postoperative adjuvant & 11 & 1 & 2 & & 14 \\
Neoadjuvant for recurrence & 1 & 1 & & 1 & 3 \\
Primary treatment for recurrence & 1 & 3 & 1 & & & 5 \\
Total & 17 & 22 & 8 & 4 & 7 & 58 \\
\hline
\end{tabular}

CCIN, corneal-conjunctival intraepithelial neoplasia; PAM, primary acquired melanosis:

SCC, squamous cell carcinoma; SGC, sebaceous gland carcinoma.

regimen was commenced 4 weeks after surgery to allow wound healing. Topical steroids and lubricants were prescribed four times a day throughout the 9-week period and beyond if required. One patient had a similar regimen but with 2 -week cycles, and two patients had a similar regimen but with 4-week cycles. All three of these variations occurred in 1998-1999 when MMC was first used in the department. A further four patients received intraoperative MMC only between 1998 and 2001, with application of $0.04 \% \mathrm{MMC}$ for $3 \mathrm{~min}$ to the exposed base and margin tissue following surgical excision. Punctual occlusion was not used during MMC treatment. All treatments were supervised by a single ocular oncologist (EK). The details of treatment indications are summarised in table 1.

Patients were examined at week 4 of treatment, and then at 1-4 weeks following completion of the course. Regular clinical review thereafter was arranged depending on the individual patient's needs. Outcome measures included initial clinical response to treatment, short-term complications, disease recurrence (including further tumour growth in lesions not showing complete regression following treatment), long-term complications (ie, complications not resolving on treatment cessation) and long-term outcomes (ie, clinical status at last review).

\section{RESULTS}

Fifty-eight patients ( 23 men and 35 women) who had received topical MMC chemotherapy were identified. The mean age at treatment was 63 years (range 24-91 years) and mean follow-up was 36 months (15 days -10 years).

Histopathological diagnosis was available in 52 patients, which confirmed the clinical diagnosis in 51 patients. One patient with a clinical diagnosis of conjunctival melanoma and treated with intraoperative MMC was found to have a conjunctival naevus on histological examination. The six patients with no histopathological diagnosis received MMC as a primary therapy for clinically diagnosed PAM.

\section{Initial clinical response}

Initial tumour response to treatment was graded as no response, partial response (a decrease in the extent/pigmentation of the lesion) or complete clinical resolution (figure 1). A favourable initial clinical response was evident in 93\% (table 2 and figure 1). Of those that showed no initial clinical response, two were PAM. One required surgical excision twice, with neoadjuvant MMC and cryotherapy to the excision margins with the second excision. The other patient defaulted follow-up then returned 5 years with increased lesion size, which was excised and revealed a benign melanocytic proliferation. One melanoma showed no initial clinical response to primary treatment with MMC for recurrence; however, due to serious co-morbidity, the patient died shortly after. Finally, one SCC showed no clinical response to excision with postoperative $\mathrm{MMC}$, thus requiring further excision with buccal mucous membrane graft to achieve disease control.

\section{Short-term complications}

Short-term complications of MMC therapy were recorded in 40 patients and are summarised in table 3 . The most common complication was allergy, $64 \%$ of which occurred during the
Figure 1 (A) Pagetoid involvement of the cornea from an upper lid sebaceous gland carcinoma. (B) The same patient showing complete clinical resolution of the corneal involvement after one cycle of topical mitomycin C (MMC), leaving only deep stromal vascularisation. (C) Primary acquired melanosis of the nasal conjunctiva. (D) The same patient showing only minimal residual pigmentation at the same site following one course of topical MMC.
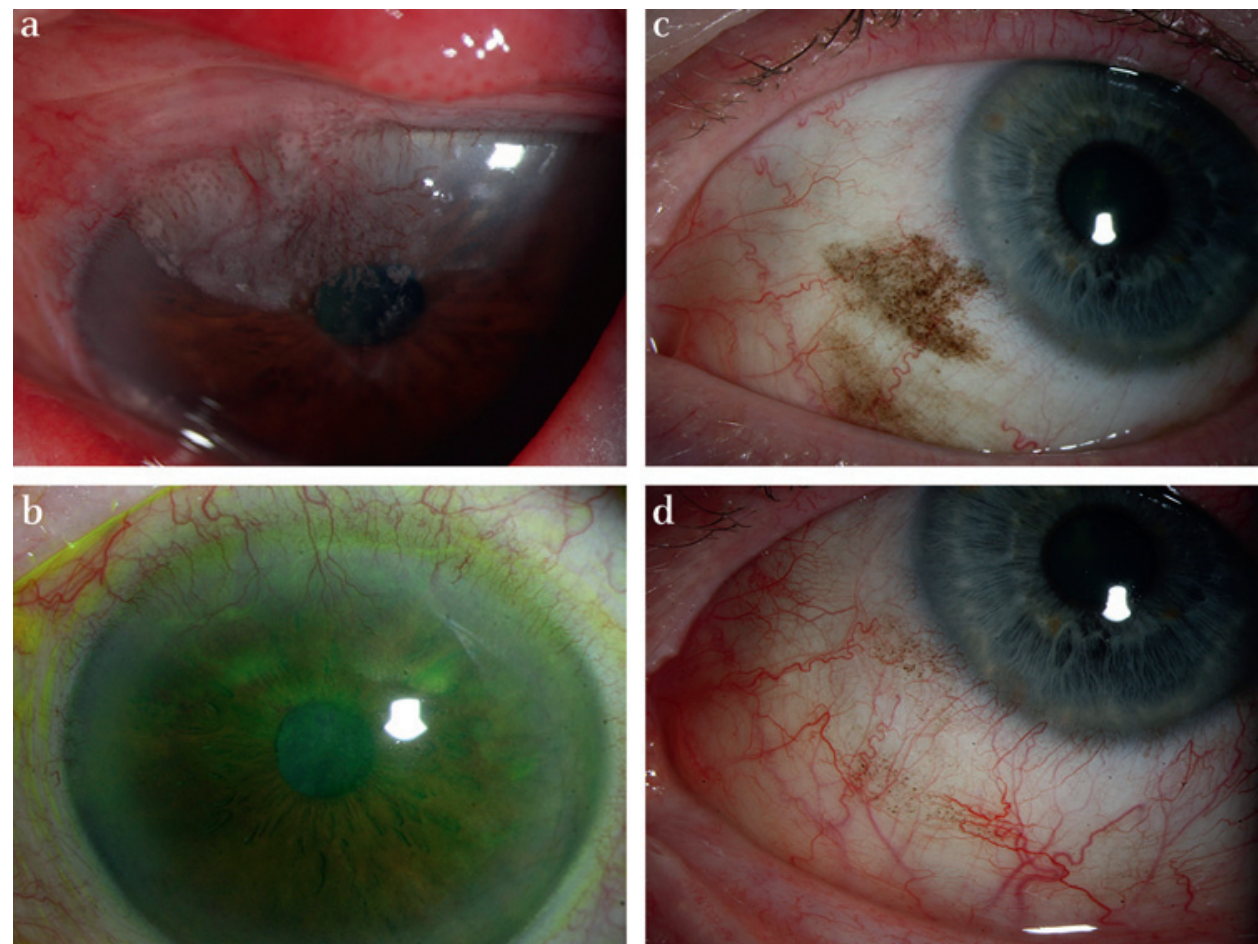
Table 2 Summary of initial clinical response and recurrence by diagnosis

\begin{tabular}{|c|c|c|c|c|c|c|c|}
\hline Clinical response & PAM & Melanoma & CCIN & SCC & SGC & Naevus & Total \\
\hline No of response & 2 & 1 & & 1 & & & 4 \\
\hline Partial & 13 & 4 & 1 & & 1 & & 19 \\
\hline Complete & 2 & 16 & 7 & 3 & 6 & 1 & 35 \\
\hline Overall recurrence & $3 / 15(20 \%)$ & $5 / 20(25 \%)$ & $0 / 7$ & $2 / 3(67 \%)$ & $4 / 7(57 \%)$ & $0 / 1$ & $14 / 53(26 \%)$ \\
\hline Mean time to recurrence (range), months & $16(14-18)$ & $14(1-30)$ & & $3.5(2-5)$ & $13.5(4-24)$ & & $12.8(1-30)$ \\
\hline $\begin{array}{l}\text { Recurrence for eyes with minimum } \\
12 \text { months follow-up }\end{array}$ & $3 / 15(23 \%)$ & $4 / 16(25 \%)$ & $0 / 4$ & $2 / 2(100 \%)$ & $4 / 5(80 \%)$ & & $13 / 42(31 \%)$ \\
\hline
\end{tabular}

2 months follow-up

CCIN, corneal-conjunctival intraepithelial neoplasia; PAM, primary acquired melanosis; SCC, squamous cell carcinoma; SGC, sebaceous gland carcinoma

For tumours treated with adjuvant MMC, complete or partial clinical response refers to overall response to both surgery and MMC. Mean times to recurrence, including the range, are included. Recurrence rates were calculated from those that showed an initial response to treatment. Overall recurrence rate was calculated from those that showed an initial response, excluding the conjunctival naevus $(n=53)$.

second 3-week period of MMC drops. Overall, $67.5 \%$ of complications were reported during the second 3-week period of MMC treatment. Despite the frequency of short-term complications, treatment cessation due to intolerance was only merited in four patients $(7 \%)$.

\section{Disease recurrence}

A total of 14 patients developed recurrent pathology at a mean interval of 12.8 months following initial treatment with MMC (table 2).

Three patients with PAM developed recurrent disease. All were treated with further topical MMC. One now has stable disease while two are being observed for clinical response following recent completion of repeat treatment.

Five patients with conjunctival melanoma developed recurrent disease (three recurred as PAM and two as melanoma). All had received treatment as a surgical adjuvant (either to surgery performed at this centre or at the referring hospital), and four had initially shown compete clinical resolution. In addition, two had stopped treatment early due to allergy. At last review, two were being observed with stable PAM, and three were clinically disease-free after additional interventions, which included further topical MMC in one and surgical excision with cryotherapy in two.

Two patients with SCC developed recurrent disease, both of whom had received postoperative adjuvant $\mathrm{MMC}$ and had initially shown complete clinical disease resolution. One patient had stopped treatment early due to allergy. Both patients were clinically disease-free at last review after additional interventions of excision with plaque brachytherapy in one, and excision, topical MMC and further excision with intraoperative MMC in the other.

Table 3 Short-term complication by symptom onset during the first or second 3-week period of topical MMC treatment

\begin{tabular}{lccl}
\hline Transient/short-term & First & Second & \\
complications & $\mathbf{3}$ weeks & $\mathbf{3}$ weeks & Total \\
\hline Hyperaemia & 2 & 6 & $8(14 \%)$ \\
Allergy/lid swelling & 4 & 7 & $11(28 \%)$ \\
Epiphora & 0 & 4 & $4(7 \%)$ \\
Discharge & 0 & 1 & $1(2 \%)$ \\
Keratoconjunctivits, mild & 4 & 4 & $8(14 \%)$ \\
Uveitis & 1 & 2 & $3(5 \%)$ \\
Corneal abrasion & 0 & 2 & $2(3 \%)$ \\
Corneal oedema & 1 & 0 & $1(2 \%)$ \\
Pyogenic granuloma & 0 & 1 & $1(2 \%)$ \\
Nosebleeds & 1 & 0 & $1(2 \%)$ \\
Total & 13 & 27 & 40 in 30 patients $(52 \%)$ \\
\hline
\end{tabular}

Of four patients with SGC who developed tumour recurrence, three had received $\mathrm{MMC}$ as a primary treatment without surgical excision. One of these was because of misreporting of the biopsy by the referring hospital as conjunctival intraepithelial neoplasia. Recurrence developed 24 months after treatment and was treated by surgical excision with skin graft; however, excision margins were involved so the patient was awaiting further excision at the time of compiling this report. The second patient received MMC as a primary treatment following biopsy confirmation of the diagnosis and showed only a partial clinical response. Further growth was evident at 4 months, which was treated by surgical excision; despite clear excision margins, further recurrence occurred almost 2 years later. The third patient developed recurrent SGC 14 months following primary treatment with topical MMC. This was treated with an additional course of MMC. Further recurrence 41 months later required exenteration. Finally, the fourth patient had neoadjuvant MMC but developed recurrence due to incomplete surgical excision. Tumour control was achieved with further surgical excision. Apart from the second patient, the tumours in the other three had initially shown complete clinical resolution.

\section{Long-term complications}

Long-term complications following topical MMC therapy occurred in a total of 19 patients (33\%). The most common complication was continuing mild keratoconjunctivitis, which was managed satisfactorily with topical lubricants in all nine affected patients. Corneal epithelial changes due to presumed limbal stem cell deficiency (LSCD) occurred in seven patients, with three suffering persistent corneal erosions (delaying further treatment with topical MMC for recurrence in one patient). Two of the seven patients had MMC as a primary treatment; thus, it is likely that MMC was the cause of this complication. The other five patients had surgical excision \pm cryotherapy in addition to MMC, making it impossible to attribute this complication solely to MMC. Epiphora due to punctal stenosis was documented in six patients, only one of whom had developed signs of allergy to the MMC drops. Finally, one patient developed lower lid ectropion following an allergic lid reaction to MMC. Several patients were also noted to have symblepharon and cicatricial conjunctival changes at the site of surgical excision, which have not been included as long-term complications of topical MMC treatment as it was felt that they were surgical complications. Overall, visual acuity was maintained within two Snellen lines in $87 \%$.

\section{DISCUSSION}

Use of topical MMC chemotherapy is now well established in the management of ocular surface neoplasia. For localised lesions, 


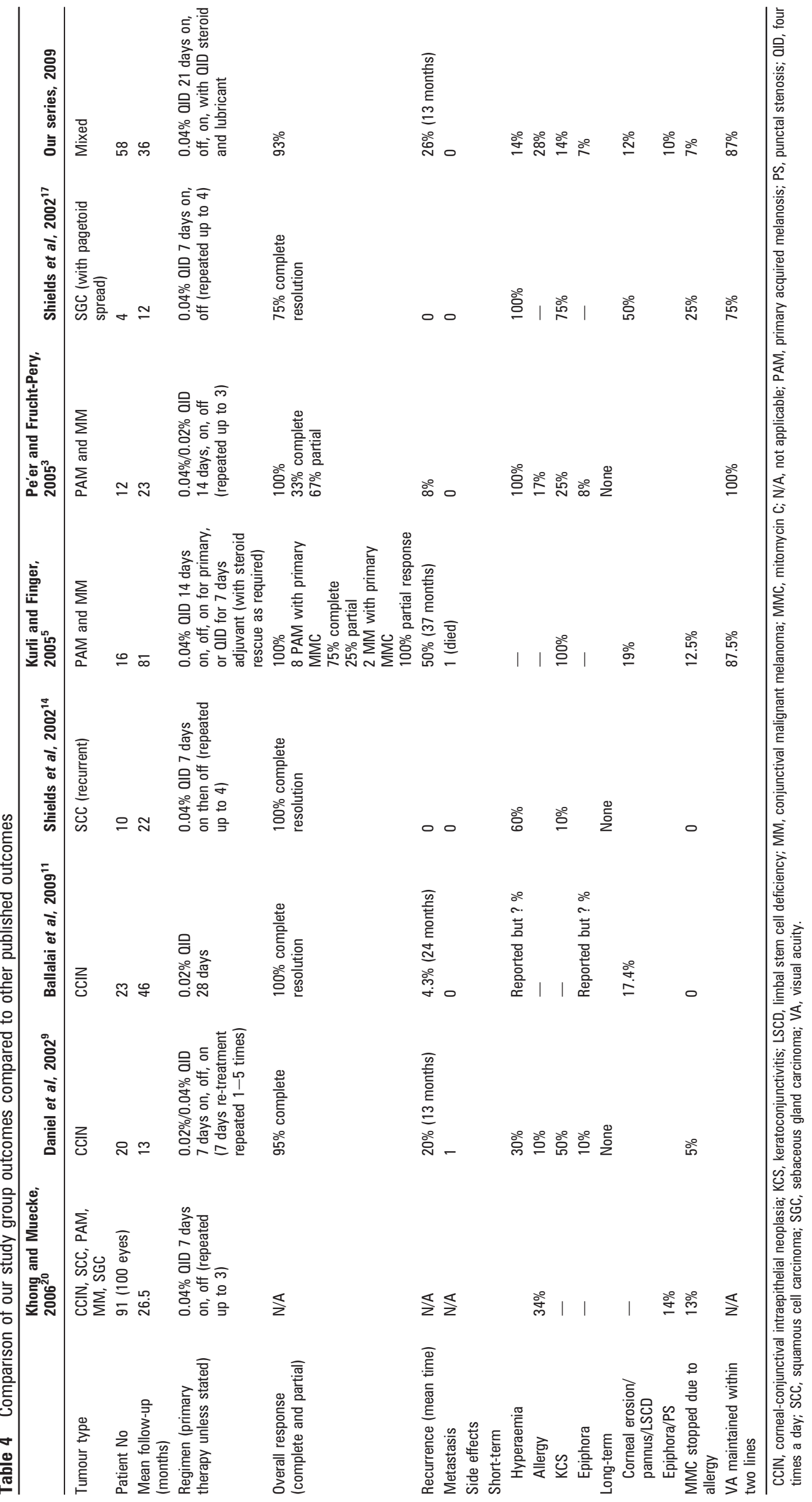


primary excision remains the treatment of choice ${ }^{24}$; however, neoadjuvant or postsurgical adjuvant treatment with MMC may further reduce the incidence of recurrence. ${ }^{51013}$ For larger lesions, neoadjuvant chemoreduction with MMC may increase the likelihood of subsequent surgical excision with clear margins, as demonstrated by one of our SGC cases that showed clear excision margins following neoadjuvant treatment. This may also reduce the amount of conjunctival resection required, thereby reducing the sequelae of extensive conjunctival resection. ${ }^{15}$

Our results confirm the highly effective role of primary $\mathrm{MMC}$ treatment in the management of both PAM and CCIN. For the other pathologies with extensive involvement, primary MMC treatment may play a role in temporising the disease, which may be particularly useful in patients with co-morbidities delaying surgery, and in those refusing radical surgery such as exenteration. This is illustrated by one of our cases of extensive SGC, in which use of primary MMC treatment delayed the need for orbital exenteration by almost 3.5 years. Primary MMC treatment was also used to treat a clinically diagnosed conjunctival melanoma in one case in our series, which demonstrated a partial response. A subsequent biopsy revealed only PAM with atypia. As no pathological diagnosis was available pretreatment, it is possible that the lesion was never invasive. Poor results with primary MMC treatment of conjunctival melanoma had been reported previously; thus, our apparently good result should be interpreted with caution. ${ }^{5}$ As seen by our results and those of others, primary $\mathrm{MMC}$ treatment is also unlikely to achieve disease control alone in SGC and SCC, particularly if the SCC is more than $4 \mathrm{~mm}$ thick. ${ }^{15}$

Toxicity to topical MMC is widely reported and remains a concern. In our series, self-limiting complications such as hyperaemia, epiphora, keratoconjunctivitis and allergy occurred frequently; however, these were only severe enough to merit treatment cessation in $7 \%$, which is much lower than reported elsewhere (table 4). The routine prescription of potent topical steroids and lubricants throughout the duration of MMC treatment and beyond if necessary, which has not been described elsewhere, may explain this low treatment drop-out rate. Of the four that required treatment cessation, two had received a longer regimen of 4 -week cycles. Three of the four patients stopped because of allergy and one because of uveitis and allergy. Uveitis as a complication of MMC has been reported previously and occurred in a further two patients in our series. Other reported short-term complications such as corneal abrasion, corneal oedema and pyogenic granuloma (requiring cryotherapy) occurred at low frequency.

Persisting complications following MMC treatment are of more concern and occurred at a higher rate than we had anticipated. The most common problem was ongoing mild keratoconjunctivitis. Although previously reported, it is likely that this minor side effect is under-reported by both patient and doctor. Persisting corneal epithelial defects and corneal irregularities due to LSCD occurred in a significant number of patients. Although a similar incidence has been reported elsewhere (table 4), many other published studies have not reported LSCD to be a problem, including an earlier report from our unit. ${ }^{25}$ However, the mean follow-up for our seven patients with LSCD was 71 months, indicating that this is likely to be a problem that becomes more common with time. As is likely in other larger tertiary referral centres, patients are often discharged back to the referring hospital for observation after a period of satisfactory disease control; thus, it is possible that our data underestimate this serious complication.
Epiphora \pm punctual stenosis occurred in $10 \%$ of patients in our series, and as reported by others, was not predicted by the development of an allergic reaction to treatment. ${ }^{2023}$ This rate is similar to that reported in the largest published series of complications of MMC treatment by Khong and Muecke, where punctal occlusion was not used. ${ }^{20}$ As for keratoconjunctivitis, it is likely that this too is under-reported by patients, as indicated by one study that found that $64 \%$ of patients had symptoms of epiphora at 1 month post treatment. ${ }^{23}$ Routine punctal occlusion is not used in our unit as it is felt that exposure of the lacrimal system to the chemotherapeutic effect of MMC is important in reducing the likelihood of tumour recurrence at this site. This may account for our incidence of punctal stenosis, but there is little evidence that this is prevented by punctal occlusion. Furthermore, punctual occlusion increases the duration of exposure of the ocular surface to MMC, which may be important in the development of intolerable side effects that were low in our series. There is also evidence that the concentration of $\mathrm{MMC}$ in the aqueous humour increases linearly with MMC concentration and exposure time, with potentially increased risk of endothelial toxicity. ${ }^{26}$ As our recurrence rates are comparable to other reported series (table 4), it is unlikely that any prolonged contact with MMC achieved with punctual occlusion is beneficial to tumour control.

In summary, the results of our series demonstrate the effective role of topical MMC in the management of a variety of ocular surface neoplasia, reducing the need for radical surgical intervention. As in other studies, short-term complications were fairly common but largely self-limiting. However, in accordance with studies with longer follow-up, limbal stem cell deficiency appears to be a significant long-term risk of MMC treatment. This serious complication should therefore be taken into consideration when contemplating use of topical MMC. In view of the malignant potential of some of these tumours, this may be an acceptable risk when compared to the consequences of previous treatment modalities.

\section{Competing interests None.}

Provenance and peer review Not commissioned; externally peer reviewed.

\section{REFERENCES}

1. Abraham LM, Dinesh S, Casson R, et al. Mitomycin: clinical applications in ophthalmic practice. Drugs 2006;66:321-40.

2. Shields CL, Demirci H, Shields JA, et al. Dramatic regression of conjunctival and corneal acquired melanosis with topical mitomycin C. Br J Ophthalmol 2002;86:244-5.

3. Pe'er J, Frucht-Pery J. The treatment of primary acquired melanosis (PAM) with atypia by topical mitomycin C. Am J Ophthalmol 2005;139:229-34.

4. Demirci H, McCormick S, Finger PT. Topical mitomycin chemotherapy for conjunctival malignant melanoma and primary acquired melanosis with atypia: clinica experience with histopathologic observations. Arch Ophthalmol 2000;118:885-91.

5. Kurli M, Finger PT. Topical mitomycin chemotherapy for conjunctival malignant melanoma and primary acquired melanosis with atypia: 12 years' experience. Graefe's Arch Clin Exp Ophthalmol 2005;243:1108-14.

6. Shields CL, Shields JA, Armstrong T. Management of conjunctival and corneal melanoma with surgical excision, amniotic membrane allograft, and topical chemotherapy. Am J Ophthalmol 2001;132:576-8.

7. Lee GA, Hirst LW. Ocular surface squamous neoplasia. Surv Ophthalmol 1995:39:429-50.

8. Fucht-Pery J, Rozenman Y. Mitomycin C therapy for corneal intraepithelial neoplasia. Am J Ophthalmol 1994;117:164-8.

9. Daniel M, Maini R, Tole D. Use of mitomycin C in the treatment of corneal conjunctival intraepithelial neoplasia. Clin Exp Ophthalmol 2002;30:94-8.

10. Chen C, Louis D, Dodd T, et al. Mitomycin C as an adjunct in the treatment of localised ocular surface squamous neoplasia. Br J Ophthalmol 2004;88:17-18.

11. Ballalai PL, Erwenne CM, Martins MC, et al. Long-term results of topical mitomycin C $0.02 \%$ for primary and recurrent conjunctival-corneal intraepithelial neoplasia. Ophthal Plast Reconstr Surg 2009;25:296-9.

12. Rozenman $\mathbf{Y}$, Frucht-Pery J. Treatment of conjunctival intraepithelial neoplasia with topical drops of mitomycin C. Cornea 2000;19:1-6. 
13. Frucht-Pery J, Rozenman Y, Pe'er J. Topical mitomycin-C for partially excised conjunctival squamous cell carcinoma. Ophthalmology 2002;109:548-52.

14. Shields CL, Naseripour M, Shields JA. Topical mitomycin C for extensive, recurrent conjunctival-corneal squamous cell carcinoma. Am J Ophthalmol 2002:133:601-6.

15. Shields CL, Demirici H, Marr BP, et al. Chemoreduction with topical mitomycin C prior to resection of extensive squamous cell carcinoma of the conjunctiva. Arch Ophthalmol 2005;123:109-13.

16. Chao AN, Shields CL, Krema H, et al. Outcome of patients with periocular sebaceous gland carcinoma with and without conjunctival intraepithelial invasion. Ophthalmology 2001;108:1877-83.

17. Shields CL, Naseripour M, Shields J, et al. Topical mitomycin-C for pagetoid invasion of the conjunctiva by eyelid sebaceous gland carcinoma. Ophthalmology 2002;109:2129-33.

18. Tumuluri K, Kourt G, Martin P. Mitomycin C in sebaceous gland carcinoma with pagetoid spread. Br J Ophthalmol 2004:88:718-19.

19. Rudkin AK, Muecke JS. Mitomycin-C as adjuvant therapy in the treatment of sebaceous gland carcinoma in high-risk locations. Clin Exp Ophthalmol 2009;37:352-6.
20. Khong JJ, Muecke J. Complications of mitomycin C therapy in 100 eyes with ocular surface neoplasia. Br J Ophthalmol 2006;90:819-22.

21. Sacu S, Segur-Eltz N, Horvat R, et al. Intumescent cataract after topical mitomycin C for conjunctival malignant melanoma. Am J Ophthalmol 2003;136:375-7.

22. Dudney BW, Malecha MA. Limbal stem cell deficiency following topical mitomycin $\mathrm{C}$ treatment of conjunctival-corneal intraepithelial neoplasia. Am J Ophthalmol 2004:137:950-1.

23. Kopp ED, Seregard S. Epiphora as a side effect of topical mitomycin C. Br J Ophthalmol 2004;88:1422-4.

24. Shields JA, Shields CL, De Potter P. Surgical management of conjunctival tumors The 1994 Lynn B. McMahon Lecture. Arch Ophthalmol 1997;115:808-15.

25. Kemp EG, Harnett AN, Chatterjee S. Preoperative topical and intraoperative local mitomycin $C$ adjuvant therapy in the management of ocular surface neoplasias. Br J Ophthalmol 2002;86:31-4.

26. Song JS, Kim JH, Yang M, et al. Mitomycin-C concentration in cornea and aqueous humor and apoptosis in the stroma after mitomycin-C application. Cornea 2007:26:461-7. 